\title{
BMJ Open Cost-effectiveness of enhancing adherence with oral bisphosphonates treatment in osteoporotic women: an empirical approach based on healthcare utilisation databases
}

\author{
Lorenza Scotti, ${ }^{1}$ Andrea Arfè, ${ }^{1}$ Antonella Zambon, ${ }^{1}$ Luca Merlino, ${ }^{2}$ Giovanni Corrao ${ }^{1}$
}

To cite: Scotti L, Arfè A, Zambon A, et al. Costeffectiveness of enhancing adherence with oral bisphosphonates treatment in osteoporotic women: an empirical approach based on healthcare utilisation databases. BMJ Open 2014:4:e003758. doi:10.1136/bmjopen-2013003758

- Prepublication history for this paper is available online. To view these files please visit the journal online (http://dx.doi.org/10.1136/ bmjopen-2013-003758).

Received 7 August 2013 Revised 10 February 2014 Accepted 13 February 2014

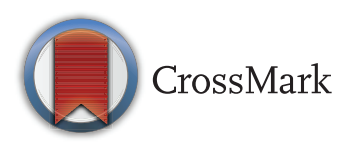

${ }^{1}$ Department of Statistics and Quantitative Methods, Unit of Biostatistics, Epidemiology and Public Health, University of Milano-Bicocca, Milan, Italy

${ }^{2}$ Operative Unit of Territorial Health Services, Lombardia Regional Council, Milan, Italy

Correspondence to Professor Antonella Zambon; antonella.zambon@unimib.it

\section{ABSTRACT}

Objective: Adherence with bisphosphonates therapy is generally low. Enhancing adherence with bisphosphonates would be effective in achieving the full benefits of therapy albeit a growth in the expenditure for supporting incremented drug use is expected. The cost-effectiveness of enhancing adherence with oral bisphosphonates in a large population of osteoporotic women has been assessed in the current study.

Design: Retrospective cohort study.

Setting: Healthcare utilisation databases of Lombardy Region, Italy.

Participants: A cohort of 28558 women aged 45 years or more, resident in the Italian Region of Lombardy, who were newly treated with oral bisphosphonates during 2003-2004, was followed for 6 years after index prescription.

Outcome measures: Fracture-free survival time, healthcare cost and incremental cost-effectiveness ratio (ICER) of enhancing adherence, that is, the additional cost that would be spent every year for gaining one fracture-free year as a consequence of enhancing adherence at a certain level.

Results: Enhanced adherence from 33\% (baseline) to $80 \%$, increased both fracture-free survivals from 970 to 973 years and healthcare costs from $€ 118000$ to $€ 265000$ every 1000 woman-years, with ICER value of $€ 53000$ (95\% Cl $€ 49000$ to $€ 58000)$. ICER values were lower for older women ( $€ 50000 ; 95 \% \mathrm{Cl}$ $€ 42000$ to $€ 58000$ ) and for those suffering from at least a chronic comorbidity ( $€ 25000 ; 95 \% \mathrm{Cl} 95 \% \mathrm{Cl}$ $€ 7000$ to $€ 47000$ ).

Conclusions: Enhancing adherence with oral bisphosphonates offers important benefits in reducing the risk of fracture, although at a substantial cost.

\section{INTRODUCTION}

Osteoporosis is a silent systemic disease characterised by low bone mineral density (BMD) and alterations of the skeleton's microarchitecture that determine fragility of

\section{Strengths and limitations of this study}

- The study was conducted in a large unselected population reflecting the patients' treatment in the real-life setting.

- A peculiarity of the current approach is that estimates are entirely based on observed data in the setting of daily clinical practice, rather than on usual pharmacoeconomic analysis.

- Residual confounding might partially affect the study results.

the bone and subsequent increased risk of fracture even in the case of mild traumas. ${ }^{1}$ With ageing populations, the burden of osteoporotic fractures on society will increase in the coming years ${ }^{2}$ and the prevention of osteoporotic fractures is therefore a major public health issue.

Randomised clinical trials (RCTs) have consistently shown that long-term treatment with oral bisphosphonates (BPs) is effective in improving BMD from $2.4 \%$ to $8.8 \%$ and reducing the risk of fracture from $12 \%$ to $49 \% .^{34}$ However, the efficacy found in RCTs may not be replicated in actual clinical practice, mainly because patients enrolled in RCTs achieve almost optimal treatment adherence, while in the clinical practice adherence is impaired. Poor adherence with BPs therapy has been observed in the real-life setting. ${ }^{5} 6$ Evidence from observational studies shows that poor adherence is associated with suboptimal improvement of bone density, ${ }^{7}$ increasing risk of fracture $e^{5-8}$ and worsening cost-effectiveness profile of therapy. ${ }^{9}$

Enhanced adherence with BPs is thereby expected to be effective in achieving full benefits from therapy. ${ }^{10}$ However, increasing expenditures for supporting increased drug use are also expected as adherence improves. 
The expenditures' impact is particularly important from a public health perspective, since it is important to optimally allocate the limited available economic resources as to maximise the population health level. ${ }^{11}$ It would therefore be suitable to evaluate cost and effectiveness of treatment jointly, in order to quantify the additional cost needed to increase the treatment's effectiveness by enhancing adherence.

Model-based economic evaluations are commonly used to compare alternative treatment strategies in osteoporosis, to support decision makers and to inform treatment guidelines. ${ }^{12-14} \mathrm{~A}$ large part of these approaches is based on data on treatment-efficacy obtained from RCTs. Although economic analyses carried out alongside RCTs have many methodological advantages, their findings may be poorly generalisable to the clinical practice. ${ }^{15}$ Other than achieving almost optimal compliance, ${ }^{5} 6$ RCTs often impose strict inclusion and exclusion criteria, while patients presenting to physicians for treatment frequently exhibit features that would exclude them from entering a trial. ${ }^{16}$ Therefore, observational studies based on high-quality data reflecting use and impact of drugs in the real world clinical practice might be alternative data sources useful for economic healthcare evaluations. ${ }^{17}$

We developed an approach to estimate the costeffectiveness profile of enhancing adherence with BPs therapy in a large population without signs of previous fracture. Costs and effectiveness were estimated from a population-based cohort study. Data were derived from the healthcare utilisation (HCU) databases of the Lombardy Region (Italy).

\section{METHODS}

\section{Setting}

The data used in the present study were retrieved from the HCU databases of Lombardy, a Region of Italy which accounts for about 16\% (9 million) of its population. In Italy, the population is fully covered by the National Health Service (NHS), which in Lombardy has been associated since 1997 with an automated system of databases collecting a variety of information, including: (1) an archive of residents receiving NHS assistance (practically the whole resident population), reporting demographic and administrative data; (2) a database on discharge diagnoses from public or private hospitals and (3) a database on outpatient drug prescriptions reimbursable by the NHS.

For each patient we linked the above databases via a single identification code. In order to preserve privacy, each identification code was automatically converted to an anonymous code. The inverse process was prevented by the deletion of the conversion table. Full details of the procedure have been reported elsewhere. ${ }^{18}$

\section{Cohort selection and follow-up}

The cohort regarded all women aged 45 years or older who received at least one prescription of oral BPs (alendronate or risedronate) between 1 January 2003 and 31 December 2004. According to current Italian regulations, these women certainly received diagnosis of osteoporosis, since BPs therapy reimbursement is obtained only for prophylaxis of osteoporotic fractures. The first prescription of BPs was defined as the index prescription.

To make the data as relevant as possible to the study aim, five categories of patients were excluded. One, patients who within 3 years before the index prescription had received BPs drug therapy, in order to ensure the inclusion of only newly treated women. Two, patients who within 3 years before the index prescription, had received drugs known to reduce the risk of bone fracture (ie, calcitonine, raloxifene and/or hormone replacement therapy (HRT)). Three, patients who within 3 years before the index prescription, had been hospitalised for the outcome of interest, to focus the data on primary prevention of fracture. Four, patients who within 3 years before the index prescription, had been hospitalised for diseases known to increase the risk of bone fracture (ie, cancer, Paget's disease, diabetes, thyroid disease, chronic renal failure and/or disease of connective tissue). Five, patients who did not reach at least 1 year of follow-up, to ensure at least 1 year of potential exposure at the drugs of interest.

Each cohort member accumulated person-years of follow-up from the date of index prescription until the earliest among the dates of outcome occurrence (ie, hospital admission for fracture) or censoring, that is, death, emigration or end of follow-up (fixed at 6 years after the date of index prescription).

\section{Exposure assessment}

Drug type, dosage, regimen and dispensation date of all BPs prescribed to each cohort member during follow-up were retrieved from the outpatient drug prescriptions database. During the study period two drug types (alendronate and risedronate), either on once daily (10 and $5 \mathrm{mg} /$ day, respectively) or once weekly (70 and $35 \mathrm{mg} /$ week, respectively) regimens, were available for reimbursement by NHS. The time span with drug available for each prescription was calculated assuming the standard frequency of intake and the prescribed dosing regimen (ie, 2 or 4 weeks), and the date of exposure ending of each dispensation was established accordingly. In this way, each day of the follow-up period was evaluated as covered or not covered by BPs availability, irrespective of whether the availability regarded single or overlapping prescriptions. Adherence was measured by dividing the cumulative number of days with BPs available for the number of days of follow-up, a measure denoted as proportion of days covered (PDC). ${ }^{19}$ A patient was considered adherent to BPs therapy if her PDC value was $80 \%$ or higher, a value conventionally used in most database studies on this topic. ${ }^{8}$

\section{Outcome ascertainment}

Hospitalisations for traumatic or pathological bone fracture (International Classification of Diseases (ICD)-9 CM: 
800-829, 733.1) occurred to cohort members during follow-up were retrieved from the hospital discharge database, and the earliest date of hospitalisation was considered as that of outcome occurrence.

\section{Additional measurements}

Several factors measurable in the HCU database of Lombardy were considered. These included age at the date of index prescription, BP type (alendronate or risedronate) and regimen (daily or weekly) filled at therapy initiation, and other drugs dispensed during follow-up (HRT, antidepressants, antidiabetic agents, lipid-lowering drugs, diuretics and $\beta$-blockers). In addition, the Charlson comorbidity index,${ }^{20}$ was calculated using diagnostic information from inpatient encounters in the 3 years prior and 1 year after the date of index prescription and expressed in two categories (ie, 0 or 1, respectively, suggesting the absence or presence of at least one comorbidity sign).

\section{Estimating the relationship between adherence and outcome}

The Cox proportional hazard regression was used to estimate the HR, and corresponding 95\% CI, for the association between adherence with BPs and time of outcome occurrence. Estimates were adjusted for time-fixed factors (age, BP type and regimen dispensed at therapy initiation and Charlson comorbidity index) as well as for factors measured during follow-up (adherence with BPs therapy and cotreatments with other drugs). As adherence, as well as all other factors measured during follow-up, can change over time, assessment of their effects requires properly accounting for the varying nature of these variables. This was carried out by fitting the Cox model that includes these factors as timedependent covariates. ${ }^{21}$ For instance, by considering the predictor variable of interest (ie, PDC categories), with this approach each participant's cumulative adherence is recalculated from the start of follow-up until the time of each outcome onset. Thus, the HR associated with a category of PDC is derived using information concurrent to the observed outcomes, rather than the adherence profile over the full length of follow-up. The same approach was used for all other time-varying factors above reported.

\section{Estimating cost and effectiveness of enhancing adherence}

Starting from the observed average PDC (baseline), three scenarios were built by progressively enhancing the adherence average level at $40 \%, 60 \%$ and $80 \%$. Healthcare costs and fracture-free survivals observed at baseline, as well as those expected for each given scenario, were computed as follows.

First, we considered the observed and expected distributions of the included patients according to their PDC values. For each scenario, a new PDC value was assigned to each patient by adding to her baseline PDC a random increment drawn from a truncated normal distribution ${ }^{22}$ with mean and variance derived from the difference between the average PDC observed at baseline and that imposed from the scenario.

Second, health outcomes were estimated by means of the years spent free from fracture observed at baseline, as well as those expected for a given scenario. Observed fracture-free survival time was computed following Neymark et al..$^{23}$ Briefly, the Breslow estimator was used to calculate the baseline hazard of each cohort member in presence of time-dependent covariates. ${ }^{24}$ The baseline hazard was combined with the parameters estimated from the Cox model to derive the survival curve of a generic cohort member, characterised by a specific history of exposure and covariate values. The years spent free from fracture by each cohort member were estimated as the area under the survival curve ${ }^{24}$ up to the maximum observed follow-up time. ${ }^{25}$ Individual survival years were then summed so resulting in the observed number of fracture-free years for every 1000 womanyears. Expected survival years were computed using the same procedure, but substituting the observed PDC value with that expected in the given scenario, keeping the values of all other covariates unchanged.

Third, healthcare annual costs observed at baseline, as well as that expected for a given scenario, were estimated. Costs from NHS perspective were calculated by the amount that the Regional Health Authority reimbursed to health providers. Costs for supporting BPs therapy, as well as those for supporting healthcare in the 1-year time horizon after index hospitalisation actually experienced by cohort members, were regarded as of interest for calculating observed cost. With the aim of providing an order of costs magnitude, the daily price for BPs therapy ranged from $€ 0.35$ to $€ 0.65$, while costs for care of a patient experiencing fracture in the first year after index hospitalisation (including direct costs for inpatients and outpatients care and assistance) ranged from $€ 2147$ for wrist fracture to $€ 14221$ for hip fracture. The same two sources were regarded for calculating expected costs. In particular, those for supporting BPs therapy at a given level of enhanced adherence (of course expected to be increased with respect to baseline) were estimated from the distribution of patients over the categories of PDC, assuming that prescription profile does not change by increasing adherence. Costs for supporting patients' healthcare experiencing fracture for a given scenario (of course expected to decrease by enhancing adherence) were estimated from the fracture-free years expected for that scenario.

Finally, we measured the incremental cost-effectiveness ratio (ICER), determined by dividing the differences in cost and health outcomes between two strategies, that is, observed at baseline versus expected according to a given scenario of enhanced adherence. ${ }^{14}$ The ICER is the additional cost that would be spent every year for gaining one fracture-free year as a consequence of enhancing adherence at a certain level. The ICER's CIs were calculated using a non-parametric bootstrap 
method based on 1000 resamples. ${ }^{26}$ The ICER was estimated for the whole cohort, as well as for strata of age at the index prescription and Charlson comorbidity score.

\section{Sensitivity analyses}

To verify the robustness of our estimates, three sensitivity analyses were performed and the corresponding ICER values were recalculated. One, alternative PDC thresholds to those used in the main analysis were considered. Two, alternative drug costs to those observed were used by substituting them with the cost of the least costly drug with the same active principle as that dispensed, as well as with the least costly drug among those available in Italian market. Three, the impact of unmeasured risk factors on adherence-outcome association was evaluated using Monte Carlo sensitivity analysis. ${ }^{27}$ To account for confounders potentially oversizing the adherenceoutcome association, we considered factors known to increase the fracture risk that also negatively affect medication adherence. Depression was identified as a possible source of such bias, since evidence exists that (1) it is a risk factor for the occurrence of bone fracture 2829 and (2) it is associated to less frequent use of health services, including poor treatment adherence. ${ }^{30}$ According to a recent meta-analysis, we assumed that depressed patients have a threefold greater risk to be non-adherent to BPs therapy compared with patients who did not suffer from depression. ${ }^{30}$ Moreover, we assumed the fracture risk to be 1.5 or 2.0 times greater in patients with depression compared to those without depression, in accordance with data from a recent meta-analysis of prospective studies examining the risk of osteoporotic fractures associated with depression, ${ }^{28}$ and findings from the US National Health and Nutrition Examination Survey. ${ }^{29}$ Following the procedure described by Steenland and Greenland, ${ }^{27}$ the adherence-fracture association was calculated after adjusting for such unmeasured confounder used to recalculate the ICER.

The SAS statistical package was used for the analyses (SAS, V.9.2). All the tested hypotheses were two-tailed, with $\mathrm{p}$ value $<0.05$ considered statistically significant.

\section{RESULTS}

\section{Cohort characteristics}

In total, 57175 women aged 45 years or older with at least a BPs prescription during 2003-2004 were identified. Among these, 24 274women were excluded because they had already received BPs or other cotreatments. Further 3273 and 1070 women were, respectively, excluded because they were affected by selected diseases, or had less than 1 year of follow-up. The remaining 28558 women were included in the cohort. They accumulated 151382 woman-years of follow-up (on average 5.3 years per patient) and 50428 woman-years of therapy (on average $33 \%$ of the time of follow-up was spent with BPs available). The cohort members who during follow-up experienced at least one hospitalisation for fracture were 1921.

Table 1 summarises selected characteristics of cohort members according to their adherence with BPs therapy during follow-up. Mean age at cohort entry was 72 years (SD 9.6 years). Alendronate administered once weekly was the most employed first-choice therapy. Overall, only $14.6 \%$ of patients were adherent with BPs therapy (ie, $\geq 80 \%$ of follow-up was covered by drug availability). Compared with women who adhered with BPs therapy, those with poor adherence were slightly younger (almost 1 year), were initially treated with alendronate administered once daily, and were less frequently cotreated with corticosteroids and more frequently with antidepressant and antidiabetic agents. There was no evidence that cotreatment with lipid-lowering drugs, diuretics and $\beta$-blockers, as well as that worse profile of comorbidity, affected adherence with BPs therapy.

\section{Relationship between adherence and outcome}

Table 2 shows that women who adhered with BPs therapy had a significant $18 \%$ reduction in fracture risk compared with those who did not adhere to the therapy. There was no evidence that either drug type or drug regimen administered at index prescription predicted the following risk of fracture. The fracture risk was significantly increased among older women who had at least a comorbidity. Cotreatment with corticosteroids, antidiabetics, lipid-lowering and antihypertensive agents were associated with a reduction in fracture risk.

\section{Cost and effectiveness of enhancing adherence}

Figure 1 shows the trends in annual cost and fracture-free survival years, as well as in ICER point and interval estimates, from baseline to scenarios of progressively enhanced adherence with BPs therapy. Progressive increase in costs (from $€ 118000$ to $€ 265000$ every 1000 woman-years) and in the time span free from fracture (from 970 to 973 years every 1000 woman-years) was observed. With respect to baseline, the annual cost that should be additionally spent to gain one fracture-free year was $€ 127000$ (95\% CI $€ 110000$ to $€ 146000$ ) when enhancing adherence at $40 \%$, but fell to $€ 53000$ (95\% CI $€ 49000$ to $€ 58000)$ when enhancing adherence at $80 \%$.

As expected, ICER values were notably lower among older women suffering of comorbidities. The ICERs calculated for the $80 \%$ adherence scenario (compared to baseline) were, respectively, €274 000 (95\% CI €240 000 to $€ 310000$ ), $€ 55000$ (95\% CI $€ 36000$ to $€ 77000$ ) and $€ 50000$ (95\% CI $€ 42000$ to $€ 58000$ ) for women aged $<60$ years, from 60 to 70 years and $\geq 71$ years. The ICER values were $€ 72000$ (95\% CI $€ 64000$ to $€ 80000$ ) and $€ 26000$ (95\% CI $€ 7000$ to $€ 47000$ ) for women without and with signs of chronic comorbidities, respectively.

\section{Sensitivity analyses}

Figure 2 shows that the use of alternative thresholds for dichotomising adherence changed to only a small extent 
Table 1 Selected traits of the included women according to their adherence with bisphosphonates therapy

\begin{tabular}{|c|c|c|c|}
\hline & \multicolumn{3}{|l|}{ Adherence category } \\
\hline & Low coverage* (N 24 383) & 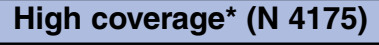 & All patients (N 28558 ) \\
\hline \multicolumn{4}{|c|}{ At the time of index prescription } \\
\hline \multicolumn{4}{|l|}{ Bisphosphonates type } \\
\hline Alendronate & $20301(86.6 \%)$ & $3153(75.5 \%)$ & $23454(82.1 \%)$ \\
\hline Risedronate & $4082(16.7 \%)$ & $1022(24.5 \%)$ & $5104(17.9 \%)$ \\
\hline Once weekly & $22265(91.3 \%)$ & $3917(93.8 \%)$ & $26182(91.7 \%)$ \\
\hline \multicolumn{4}{|l|}{ Cotreatments } \\
\hline Corticosteroids & $9017(37.0 \%)$ & $1674(40.1 \%)$ & $10691(37.4 \%)$ \\
\hline Antidepressants & $9261(38.0 \%)$ & $1417(34.0 \%)$ & $10678(37.4 \%)$ \\
\hline Drugs used for diabetes & $1777(7.3 \%)$ & $262(6.3 \%)$ & $2039(7.1 \%)$ \\
\hline$\geq 1$ & $4207(17.3 \%)$ & $717(17.2 \%)$ & $4924(17.2 \%)$ \\
\hline
\end{tabular}

the cost-effectiveness estimates associated with enhancing adherence from baseline to $80 \%$ (being the ICER values $€ 52000$ and $€ 68000$ for thresholds of $70 \%$ and $90 \%$, respectively). The ICER values associated with an $80 \%$ adherence scenario would be reduced to $€ 46000$ or $€ 34000$ if all patients were treated with the less costly drug within each active principle, or the less costly drug among those available in Italian market. Finally, the ICERs changed only to a small extent by external adjustment for depression status, even imposing the strongest confounder-outcome association (the ICERs being $€ 72000$ and $€ 95000$ when imposing that fracture risk was
1.5 and 2.0 times greater in exposed than in unexposed to the unmeasured confounder, respectively).

\section{DISCUSSION}

In our population, nearly 15000 women aged 45 years or older started BPs therapy every year (almost 66 new users every 10000 woman-years). According to both Italian regulations and the adopted exclusion criteria, these women must have received diagnosis of osteoporosis and must have started therapy for primary prevention of bone fracture. On an average, the cost for drug

Table 2 Adjusted HRs, and corresponding 95\% Cls, for the association between selected traits of the included women, including their adherence with bisphosphonates therapy during follow-up, and the risk of fracture

\begin{tabular}{|c|c|c|c|}
\hline & Number of fractures & Adjusted HR* & $95 \% \mathrm{Cl}$ \\
\hline Age in years & & 1.07 & 1.07 to 1.08 \\
\hline \multicolumn{4}{|l|}{ Therapy with bisphosphonates } \\
\hline \multicolumn{4}{|l|}{ At index prescription } \\
\hline Risedronate vs alendronate & 355 vs 1566 & 0.89 & 0.78 to 1.00 \\
\hline Once weekly vs once daily & 1755 vs 166 & 0.97 & 0.82 to 1.15 \\
\hline \multicolumn{4}{|l|}{ Adherence during follow-up } \\
\hline High vs low coverage & 344 vs 1577 & 0.82 & 0.73 to 0.93 \\
\hline \multicolumn{4}{|l|}{ Cotreatments (yes vs no) } \\
\hline Corticosteroids (users vs no users) & 514 vs 1407 & 0.65 & 0.58 to 0.72 \\
\hline Antidepressants (users vs no users) & 744 vs 1177 & 1.02 & 0.93 to 1.12 \\
\hline Drugs used for diabetes (users vs no users) & 107 vs 1814 & 0.82 & 0.67 to 0.99 \\
\hline Lipid-lowering agents (users vs no users) & 353 vs 1568 & 0.59 & 0.52 to 0.66 \\
\hline Diuretics or $\beta$-blockers (users vs no users) & 896 vs 1025 & 0.72 & 0.65 to 0.78 \\
\hline \multicolumn{4}{|l|}{ Comorbidity } \\
\hline Charlson score ( $\geq 1$ vs 0 ) & 482 vs 1439 & 1.83 & 1.65 to 2.04 \\
\hline
\end{tabular}

Healthcare utilisation database of Lombardy, Italy, 2003-2010.

*HR estimated by fitting Cox proportional hazard model. Estimates are adjusted for all the factors reported in the table (see text). 
Figure 1 Trends in healthcare cost and fracture-free survival (upper box) and in incremental cost-effectiveness ratio, with corresponding $95 \%$ Cls (below box), from baseline to scenarios of progressively enhancing adherence with bisphosphonates therapy. Healthcare utilisation database of Lombardy, Italy, 2003-2010.

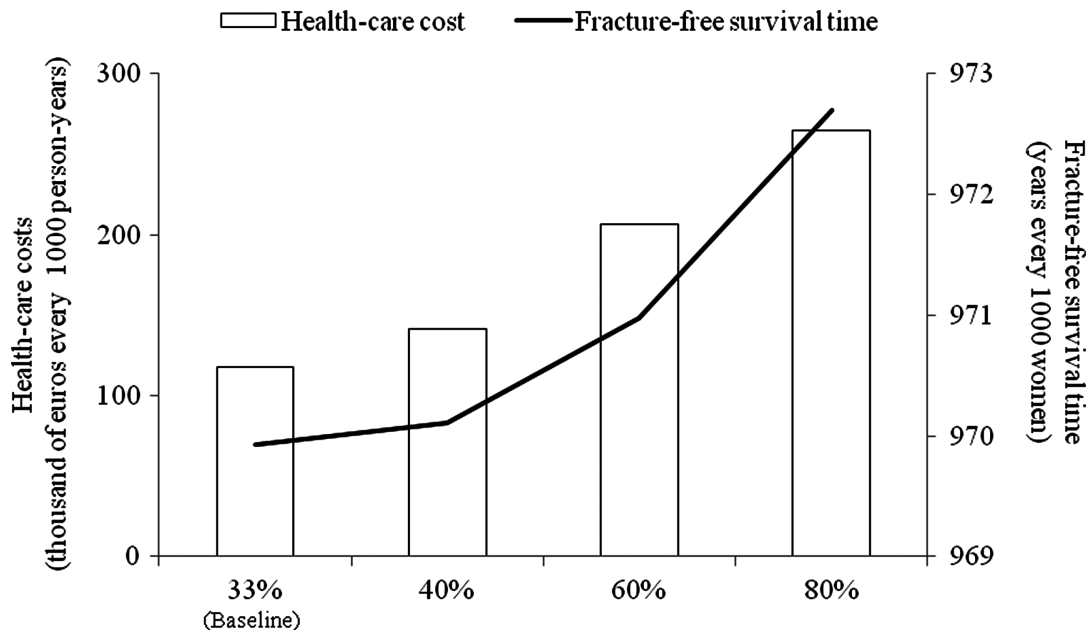

Average adherence level

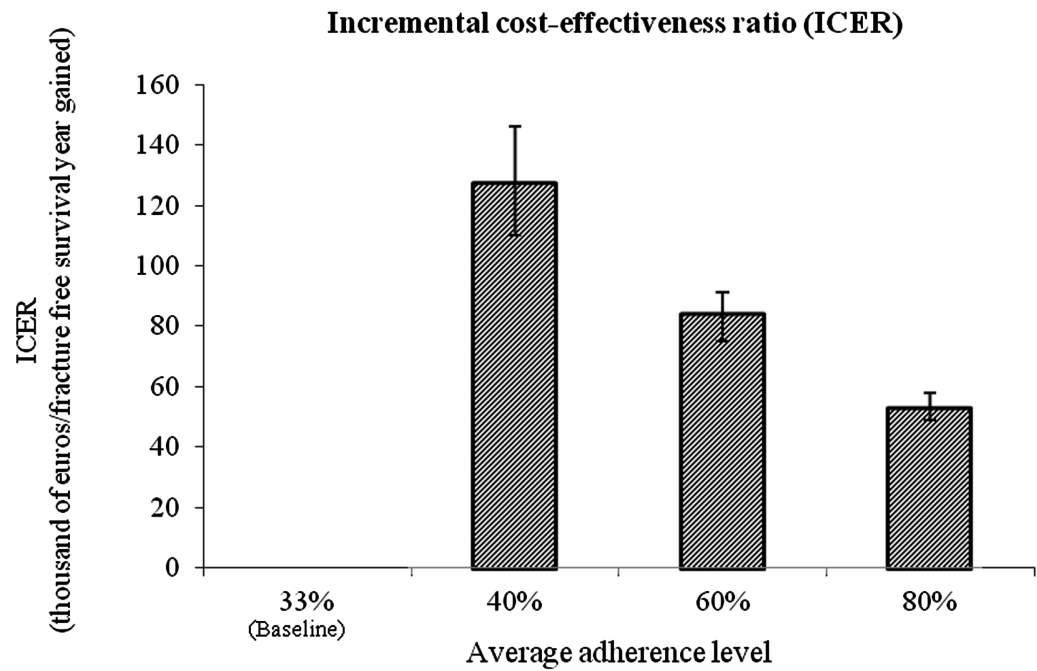

acquisition amounted to about $€ 118000$ every 1000 woman-years (ie, an annual cost of $€ 1.7$ million for the entire cohort of BP-therapy initiators). Drug cost, however, strongly depends on the average BP-therapy adherence level observed in the considered population. With respect to other reports, ${ }^{5} 6$ a very low adherence level was found in our setting, since only $33 \%$ of the observational period was covered by drug availability. Consistently with others, ${ }^{5-8}$ we found that, compared with women with adherence level $<80 \%$, those with higher adherence had an $18 \%$ reduced risk of fracture. This implies that should higher adherence have occurred in the population, a lower number of women would have experienced hospitalisation for bone fracture and a gain in fracture free survival time would have been observed. Based on our estimates, 0.18 or 2.77 fracture-free years are, respectively, expected to be gained every 1000 woman-years at risk for scenarios involving average adherence of $40 \%$ or $80 \%$ in the target population. However, €22 000 (or $€ 146000$ ) every 1000 woman-years are expected to be additionally spent with these scenarios. Thus, as a balance, we estimated that by enhancing average adherence level from baseline to $40 \%$ (or $80 \%$ ), $€ 127000$ (or $€ 53000$ ) would be additionally spent to gain one fracture-free survival year. Additional costs of $€ 51000$ and $€ 26000$ are, respectively, expected by enhancing adherence from baseline to $80 \%$ among older women and among those suffering of comorbidities.

Such estimates should be compared with those supported by the NHS for taking care of patients who experience bone fracture during the first year after the first hospitalisation. Rough estimates may be made by dividing the annual direct costs sustained for hospitalisations and rehabilitation following bone fractures by the number of patients who every year were hospitalised for fracture. Estimates of this type may be drawn out by recent papers from Italy, France and Canada that, respectively, reported annual cost per patient of $€ 11000$, $€ 12000$ and $€ 40000$. $^{31-33}$

This study is unique in several respects. Results from meta-analysis of RCTs have been extensively used to model cost-effectiveness of the treatment of osteoporosis. $^{34}$ There are, however, circumstances where 
ICER value from main analyis

$\operatorname{ICER}(95 \% \mathrm{CI})$

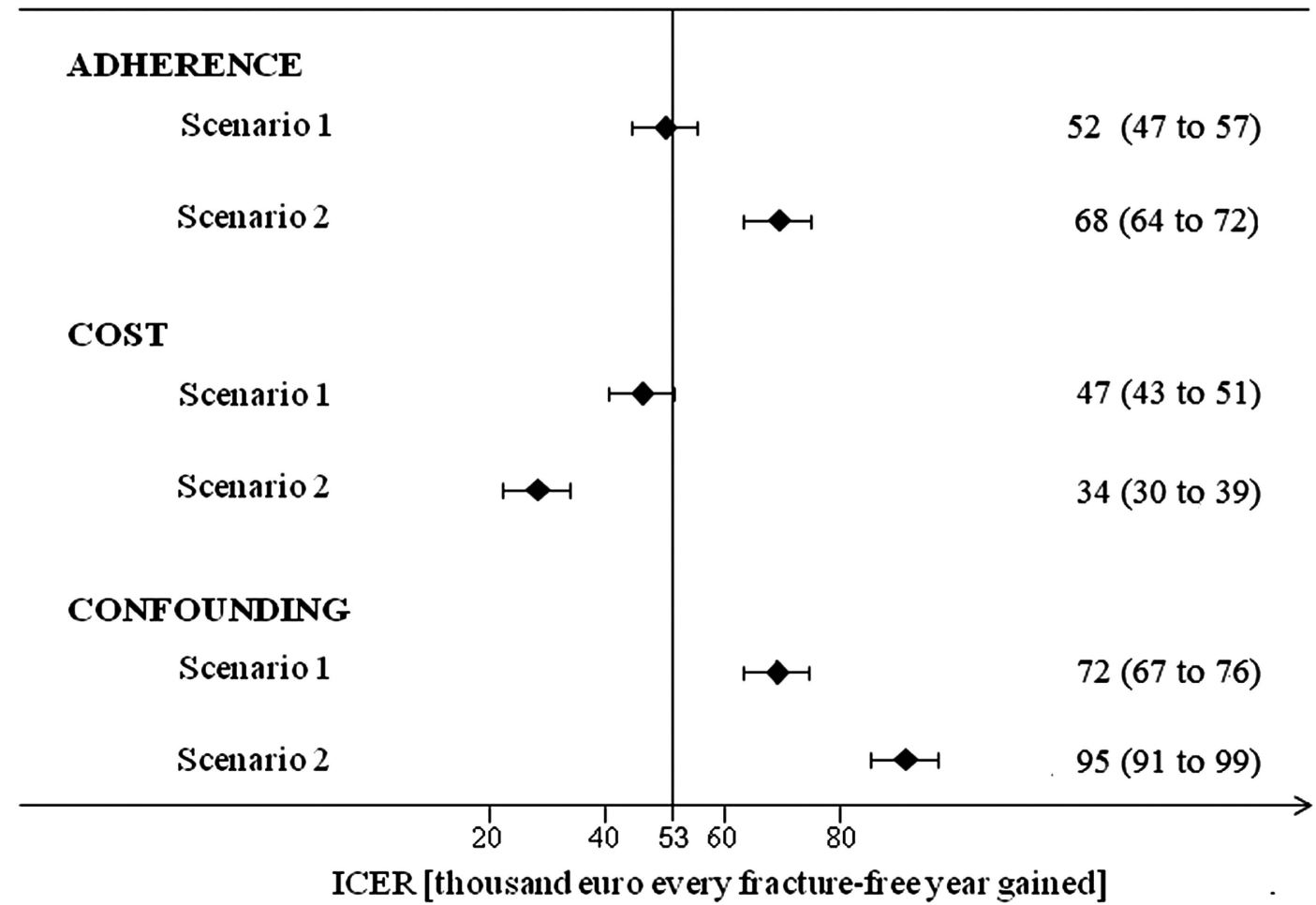

Figure 2 Influence of choosing PDC (proportion of days covered by bisphosphonates (BPs) availability) threshold for dichotomising adherence category (A; see ADHERENCE footnote), spending for drug therapy with BPs (B; see COST footnote), and controlling for unmeasured confounders (C; see CONFOUNDING footnote) on the incremental cost-effectiveness ratio values associated with enhanced adherence from baseline to $80 \%$. Healthcare utilisation database of Lombardy, Italy, 20032010. ADHERENCE-in the main analysis a patient was considered adherent with therapy if her PDC value was $80 \%$ or higher. We reported alternative thresholds of $70 \%$ and $90 \%$, respectively, denoted first and second scenario. COST—in the main analysis the cost of BPs truly dispensed in the clinical practice was considered. We here considered alternative cost including the less costly drug within each therapeutic class as that dispensed (first scenario), and the less costly drug among those available in Italian market (second scenario). CONFOUNDING -in the main analysis the extent of adherence-outcome association was adjusted for factors directly measured from databases. We here used external adjustment imposing that (1) compared with patients unexposed to the unmeasured confounder, those exposed have a three times greater risk to be no adherent with BPs therapy; (2) the risk of fracture was 1.5 (first scenario) and 2.0 (second scenario) times greater in exposed than in unexposed to the unmeasured confounder.

randomisation is not possible, such as in studies aimed at investigating the impact of treatment adherence on clinical outcomes in the 'real-life' setting. A first peculiarity of our approach is that estimates are entirely based on observed data in the setting of daily clinical practice, rather than on Markov microsimulation models typical of economic analysis. ${ }^{35}$ Assuming that a simulation model is only necessary when direct observation of the phenomenon is not available or cannot be collected ${ }^{17}$ our approach provides an original framework for evaluating the cost-effectiveness of enhancing adherence entirely based on HCU databases. A second peculiarity of our approach is that the increase of fracture-free survival time was used to measure effectiveness of enhancing adherence. This approach, although unusual, was described as essential for economic evaluations of healthcare interventions for which patient survival is the principal outcome. ${ }^{22}$ Notwithstanding these peculiarities, our estimates were of the same order of magnitude with respect to those using more usual approaches. For instance, a recent microsimulation study reported that the costs per QALYs gained, for branded BPs were estimated at $€ 19000, € 32000$ and $€ 64000$ for medical possession rates of $100 \%, 80 \%$ and $60 \%$, respectively. ${ }^{36}$

In Italy: (1) a cost-free uniformly organised healthcare system involves practically all citizens, (2) the healthcare system is managed by a set of databases including inpatients hospital discharge and outpatients drug prescription data, (3) these databases guarantee universal coverage of population receiving health assistance from NHS and (4) quality of these databases has been repeatedly ascertained, although in a clinical setting different from that considered in the current study. ${ }^{37}$ Owing to these characteristics, the complete history of health services utilisation of a very large unselected population is 
available and real world clinical practice, including health and economic burden of medical interventions, may be investigated. According to our best knowledge, the current study offers unique insight on the potentials of HCU databases to estimate the cost-effectiveness profile of medical interventions on osteoporosis.

A number of sensitivity analyses allowed us to verify the robustness of our findings. For example, the use of alternative PDC categorisations as well as adjustment for unmeasured confounders did not substantially modify the reported estimates. We also showed that the cost-effectiveness profile was greatly improved by considering less costly drugs, typically generic drugs. Of course, this is true under the assumption that treatment with generic drugs does not lead to lower adherence or more fracture-related hospitalisations compared with brand-name therapy.

Our study has a number of potential limitations. External validity is a first major concern. Drug costs are extremely variable among countries, and will likely decrease with increasing competition from generics. ${ }^{38}$ As a consequence, our estimates cannot be universally generalised.

Both the components of cost-effectiveness estimate are likely affected by sources of systematic uncertainty. As far as costs are concerned, our analysis did not include those to support interventions aimed at improving adherence. Although interventions that try to change the adherence level in population can be very costly and the objective is very hard to obtain, ${ }^{39-41}$ we were not able to measure or even approximate costs for supporting interventions aimed at improving adherence since, to our best knowledge, no studies have been performed on this topic in Italy. Furthermore, interventions able to enhance adherence unlikely have an effect only on one therapeutic class, so that their cost should impact on a variety of chronic therapies. This implies that our study must be regarded as aimed to investigate the costeffectiveness of enhanced adherence, rather than the cost-effectiveness profile of interventions aimed at improving drug adherence. In the past few years a substantial improvement of adherence with chronic drug therapies has been noticed in Lombardy Region of Italy, though no specific interventions were implemented in this regard. This suggests that a natural experiment is in progress in our setting, and our estimates can help us to understand the order of magnitude of costs and effectiveness expected from these 'unplanned' changes.

Regarding the effectiveness measure, different sources of uncertainty in the measurement of the outcomeadherence relationship should be carefully considered. As the data on the outcome onset were drawn from the archive of hospitalised patients, our conclusions only concern fractures treated on an inpatients basis. This issue is particularly important with respect to vertebral fractures, the majority of which are not hospitalised in most countries. The inclusion of these would have significantly decreased the estimated cost per fracture-free year gained.
Measurement errors due to inaccuracies in the identification of therapeutic and diagnostic codes might also have occurred. Furthermore, in our study adherence with treatment was derived from drug prescriptions, which is the most feasible and widely used method to estimate it in large populations. ${ }^{42}$ With this method, however, it must be assumed that the PDC by a prescription corresponds to the proportion of days of drug use, which may not be invariably the case. As exposure misclassification is expected to be independent from the disease status, the reduction of fracture risk associated with high levels of adherence might be larger than that quantified by our study ${ }^{43}$ and consequently, the adherence-enhancing impact should become more cost effective.

As the allocation to adherence levels was not randomised in our study, the results may be affected by confounding. Notwithstanding this, we attempted to limit confounding by adjusting estimates for available demographic, therapeutic and clinical factors, and by external adjustment for unmeasured confounders, higher adherence could still be a surrogate for other unmeasured characteristics. For example, evidence exists that patients with severe osteoporosis appear to be more adherent with BP therapy. ${ }^{44}{ }^{45}$ This suggests that the reduction of fracture risk associated with high adherence might be larger, and enhanced adherence should become more cost effective, than that quantified by our study. Other factors might exert stronger confounding effect on the relation of interest. For instance, patients with cognitive impairment, those with family history of hip fracture and current smokers, may have poorer adherence and worse outcomes.

Finally, two further weaknesses need to be emphasised. One, in the current application, our study estimates only concern the time span along which data are available (ie, the 6-year period of follow-up). Two, our implicit assumption of steady effect of the adherence over the entire period of follow-up may be unrealistic. This suggests that additional methodological work is needed for refining our approach.

All these limitations taken together, indicate that costeffectiveness profile obtained by our approach may lead to overestimation (not capturing outpatient fractures and those who are adherent likely having high risk of fracture at baseline), as well as to underestimation of costs (mainly those of intervention to improve adherence). For this reason, in the current form our approach only indicates the direction, rather than the exact magnitude, of trends in costs and effectiveness along scenarios of enhanced adherence.

In conclusion, the present study suggests that our approach based on HCU databases is useful to explore the cost-effectiveness of enhancing adherence with chronic therapies. We found that the impact of enhanced adherence offers important benefits in reducing the fracture risk but at a substantial cost. However, proper treatment strategies (eg, increasing adherence 
monitoring of women at high risk of fracture and dispensing drugs with cheapest costs), are likely to be associated with added value for the healthcare system.

Contributors GC generated the initial study idea, designed the study and drafted the manuscript with LS. LS and AA performed the data analysis. AZ provided statistical support. LM extracted the data and authorised their utilisation. All authors assisted the results interpretation and manuscript revision.

Funding This study was supported by the Italian Minister for University and Research ("Fondo d'Ateneo per la Ricerca" portion, year 2011).

Competing interests None.

Provenance and peer review Not commissioned; externally peer reviewed.

Data sharing statement No additional data are available.

Open Access This is an Open Access article distributed in accordance with the Creative Commons Attribution Non Commercial (CC BY-NC 3.0) license, which permits others to distribute, remix, adapt, build upon this work noncommercially, and license their derivative works on different terms, provided the original work is properly cited and the use is non-commercial. See: http:// creativecommons.org/licenses/by-nc/3.0/

\section{REFERENCES}

1. NIH Consensus Development Panel on Osteoporosis Prevention, Diagnosis, and Therapy. Osteoporosis prevention, diagnosis, and therapy. JAMA 2001;285:785-95.

2. Cashman KD. Diet, nutrition, and bone health. J Nutr 2007:137:2507S-12S.

3. Black DM, Cummings SR, Karpf DB, et al. Randomised trial of effect of alendronate on risk of fracture in women with existing vertebral fractures. Fracture Intervention Trial Research Group. Lancet 1996;348:1535-41.

4. Reginster J, Minne HW, Sorensen $\mathrm{OH}$, et al. Randomized trial of the effects of risedronate on vertebral fractures in women with established postmenopausal osteoporosis. Vertebral Efficacy with Risedronate Therapy (VERT) Study Group. Osteoporos Int 2000;11:83-91.

5. Siris ES, Harris ST, Rosen CJ, et al. Adherence to bisphosphonate therapy and fracture rates in osteoporotic women: relationship to vertebral and nonvertebral fractures from 2 US claims databases. Mayo Clin Proc 2006;81:1013-22.

6. Downey TW, Foltz SH, Boccuzzi SJ, et al. Adherence and persistence associated with the pharmacologic treatment of osteoporosis in a managed care setting. South Med J 2006;99:570-5.

7. Yood RA, Emani S, Reed JI, et al. Compliance with pharmacologic therapy for osteoporosis. Osteoporos Int 2003;14:965-8.

8. Blouin J, Dragomir A, Moride Y, et al. Impact of noncompliance with alendronate and risedronate on the incidence of nonvertebral osteoporotic fractures in elderly women. Br J Clin Pharmacol 2008:66:117-27.

9. Mullins $C D$, Ohsfeldt RL. Modelling the annual costs of postmenopausal prevention therapy: raloxifene, alendronate, or estrogen-progestin therapy. J Manag Care Pharm 2003;9:150-8.

10. McDonald HP, Garg A, Haynes RB. Interventions to enhance patient adherence to medication prescriptions: scientific review. JAMA 2002;288:2868-79.

11. Drummond MF, O'Brien B, Stoddart GL, et al. Methods for the economic evaluation of health care programmes. 2nd edn. New York: Oxford University Press, 1997.

12. Ström O, Borgström F, Sen SS, et al. Cost-effectiveness of alendronate in the treatment of postmenopausal women in 9 European contries-an economic evaluation based on the fracture intervention trial. Ostoporos Int 2007;18:1047-61.

13. Cotté FE, Fautrel B, De Pouvourville G. A Markov model simulation of the impact of treatment persistence in post-menopausal osteoporosis. Med Decis Making 2009;29:125-39.

14. Patrick A, Schousboe JT, Losina E, et al. The economics of improving medication adherence in osteoporosis: validation and application of a simulation model. J Clin Endocrinol Metab 2011;96:2762-70.

15. Simon G, Wagner E, Vonkorff M. Cost-effectiveness comparisons using "real world" randomized trials: the case of new antidepressant drugs. J Clin Epidemiol 1995;48:363-73.

16. Rothwell PM. External validity of randomised controlled trials: "To whom do the results of this trial apply?" Lancet 2005;365:82-93.

17. Sheldon TA. Problems of using modelling in the economic evaluation of health care. Health Econ 1996;5:1-11.
18. Corrao G, Cesana G, Merlino L. Pharmacoepidemiological research and the linking of electronic health care databases available in the Italian region of Lombardy. BioMed Stat Clin Epidemiol 2008:2:117-25.

19. Peterson AM, Nau DP, Cramer JA, et al. A checklist for medication compliance and persistence studies using retrospective databases. Value Health 2007;10:3-12.

20. Deyo RA, Cherkin DC, Ciol MA. Adapting a clinical comorbidity index for use with ICD-9-CM administrative databases. J Clin Epidemiol 1992;45:613-19.

21. Marubini E, Valsecchi MG. Analysing survival data from clinical trials and observational studies. New York: John Wiley \& Sons, 1995.

22. Forbes $\mathrm{C}$, Evans M, Hastings N, et al. Statistical distributions. 4th edn. Hoboken: John Wiley \& Sons, 2011.

23. Neymark N, Adriaenssen I, Gorlia T, et al. Estimating survival gain for economic evaluations with survival time as principal endpoint: a cost-effectiveness analysis of adding early hormonal therapy to radiotherapy in patients with locally advanced prostate cancer. Health Econ 2002;11:233-48.

24. Lin DY. On the Breslow estimator. Lifetime Data Anal 2007;13:471-80.

25. Karrison T. Use of Irwin's restricted mean as an index for comparing survival in different treatment groups-interpretation and power considerations. Controlled Clin Trials 1997;18:151-67.

26. Wang $\mathrm{H}$, Zhao $\mathrm{H}$. A study on confidence intervals for incremental cost-effectiveness ratios. Biom J 2008;50:505-14.

27. Steenland K, Greenland S. Monte Carlo sensitivity analysis and Bayesian analysis of smoking as an unmeasured confounder in a study of silica and lung cancer. Am J Epidemiol 2004;160:384-90.

28. Wu Q, Liu J, Gallegos-Orozco JF, et al. Depression, fracture risk, and bone loss: a meta-analysis of cohort studies. Osteoporos Int 2010;21:1627-35.

29. Mussolino ME. Depression and hip fracture risk: the NHANES I epidemiologic follow-up study. Public Health Rep 2005;120:71-5.

30. DiMatteo MR, Lepper HS, Croghan TW. Depression is a risk factor for noncompliance with medical treatment. Meta-analysis of the effects of anxiety and depression on patient adherence. Arch Intern Med 2000;160:2101-7.

31. Piscitelli P, Gimigliano F, Gatto S, et al. Hip fractures in Italy: 20002005 extension study. Osteoporos Int 2010;21:1323-30.

32. Maravic M, Jouaneton $B$, Vainchtock $A$, et al. Economic burden of osteoporosis in women: data from the 2008 French hospital database (PMSI). Clin Exp Rheumatol 2012;30:222-7.

33. Tarride JE, Hopkins RB, Leslie WD, et al. The burden of illness of osteoporosis in Canada. Osteoporos Int 2012;23:2591-600.

34. Hiligsmann $\mathrm{M}$, Rabenda $\mathrm{V}$, Bruyère $\mathrm{O}$, et al. The clinical and economic burden of non-adherence with oral bisphosphonates in osteoporotic patients. Health Policy 2010;96:170-7.

35. Briggs A, Sculpher M. An introduction to Markov modeling for economic evaluations. Pharmacoeconomics 1998;13:397-409.

36. Hiligsmann M, Rabenda V, Gathon HJ, et al. Potential clinical and economic impact of nonadherence with osteoporosis medications. Calcif Tissue Int 2010;86:202-10.

37. Corrao G, Parodi A, Zambon A, et al. Reduced discontinuation of antihypertensive treatment by two drug combination as 1st step. Evidence from daily life practice. $J$ Hypertens 2010;28:1584-90.

38. Danzon PM, Furukawa MF. International prices and availability of pharmaceuticals in 2005. Drugs spending differences reflect availability and use, not just prices. Health Aff 2011;27:221-33.

39. Gleeson T, Iversen MD, Avorn J, et al. Interventions to improve adherence and persistence with osteoporosis medications: a systematic literature review. Osteoporos Int 2009;20:2127-34

40. Chapman RH, Ferrufino CP, Kowal SL, et al. The cost and effectiveness of adherence-improving interventions for antihypertensive and lipid-lowering drugs. Int J Clin Pract 2010;64:169-81.

41. Solomon $\mathrm{DH}$, Iversen MD, Avorn $\mathrm{J}$, et al. Osteoporosis telephonic intervention to improve medication regimen adherence: a large, pragmatic, randomized controlled trial. Arch Intern Med 2012;172:477-83.

42. Fitz-Simon N, Bennett K, Feely J. A review of studies of adherence with antihypertensive drugs using prescription databases. Ther Clin Risk Manag 2005;1:93-106.

43. Copeland KT, Checkoway H, McMichael AJ, et al. Bias due to misclassification in the estimation of relative risk. Am J Epidemiol 1997; 105:488-95.

44. Pressman A, Forsyth B, Ettinger B, et al. Initiation of osteoporosis treatment after bone mineral density testing. Osteoporos Int 2001;12:337-42.

45. Rossini M, Bianchi G, Di Munno O, et al. Determinants of adherence to osteoporosis treatment in clinical practice. Osteoporos Int 2006;17:914-21. 